\title{
OLAW issues guidance on the use of telecommunications for IACUC meetings
}

On 24 March 2006, the Office of Laboratory Animal Welfare (OLAW), Office of Extramural Research, issued new guidance on the use of telecommunications for IACUC meetings ${ }^{1}$. The purpose of this timely and very helpful guidance is to permit Public Health Service (PHS) awardee institutions to conduct official IACUC business requiring a quorum, with telephone or video conferencing, provided the specific criteria described below are met. Until now, sections IV.C.2 and 6 of the PHS Policy on Humane Care and Use of Laboratory Animals (PHS Policy) ${ }^{2}$ required a convened meeting of a quorum of the IACUC to approve a proposed research project or suspend an activity involving animals.

In similar guidance issued on $28 \mathrm{March}$ 2000, Thomas Puglisi, Director, Division of Human Subject Protections, Office for Protections from Research Risks (OPRR) wrote regarding Institutional Review Boards (IRBs), that “... OPRR will recognize as 'convened' those IRB meetings conducted via telephone conference call, provided that each participating IRB member (i) has received all pertinent material prior to the meeting, and (ii) can actively and equally participate in the discussion of all protocols 3 ."

The following are conditions that IACUCs must follow when using methods of telecommunications to conduct official IACUC business that requires a quorum:

- "All members are given notice of the meeting.

- "Documents normally provided to members during a physically-convened meeting are provided to all members in advance of the meeting.

- "All members have access to the documents and the technology necessary to fully participate.
- "A quorum of voting members is convened when required by PHS Policy.

- "The forum allows for real time verbal interaction equivalent to that occurring in a physically-convened meeting (i.e., members can actively and equally participate and there is simultaneous communication).

- "If a vote is called for, the vote occurs during the meeting and is taken in a manner that ensures an accurate count of the vote. A mail ballot or individual telephone polling cannot substitute for a convened meeting.

- "Opinions of absent members that are transmitted by mail, telephone, fax or e-mail may be considered by the convened IACUC members but may not be counted as votes or considered as part of the quorum.

- "Written minutes of the meeting are maintained in accord with the PHS Policy, IV.E.1.b. ${ }^{\text {" }}$

According to this announcement, the United States Department of Agriculture, Animal and Plant Health Inspection Service, Animal Care (USDA, APHIS, AC), which enforces the Animal Welfare Act Regulations agrees with this Notice and will publish consistent guidance in its Research Facility Inspection Guide.

1. Office of Laboratory Animal Welfare, Office of Extramural Research. Guidance on Use of Telecommunications for IACUC Meetings under the PHS Policy on Humane Care and Use of Laboratory Animals. Notice Number: NOT-0D-06052. (24 March 2006). http://grants.nih.gov/ grants/guide/notice-files/NOT-0D-06-052.html.

2. Public Health Service. Policy on Humane Care and Use of Laboratory Animals IV.C.2, IV.C.6 (US Department of Health and Human Services, Washington, DC, 1986, revised 2002).

3. Director, Division of Human Subject Protections, OPRR. IRB Meetings Convened via Telephone Conference Call. (28 March 2000). http://www. hhs.gov/ohrp/references/irbtel.pdf. 